\title{
BIBLIOGRAPHY
} 106.

1. M. G. Barratt, Truck groups. I, Proc. London Math. Soc. vol. 5 (1955) pp. 71-

2. R. Bott, The stable homotopy of the classical groups, Ann. of Math. vol. 70 (1959) pp. 313-337.

3. I. M. James, The intrinsic join: a study of the homotopy groups of Stiefel manifolds, and Cross-sections of Stiefel manifolds, Proc. London Math. Soc. vol. 8 (1958) pp. 507-636 and pp. 536-547.

4. - Whitehead products and vector fields on spheres, Proc. Cambridge Philos. Soc. vol. 53 (1957) pp. 817-820.

5. H. Toda, Composition methods in homotopy groups of spheres, to be published in Annals of Mathematics Studies, Princeton University.

6. G. W. Whitehead, A generalization of the Hopf invariant, Ann. of Math. vol. 51 (1950) pp. 192-237.

7. J. H. C. Whitehead, On the groups $\pi_{r}\left(V_{n, m}\right)$ and sphere-bundles, Proc. London Math. Soc. vol. 48 (1944) pp. 243-291.

The Institute for Advanced Study and UNIVERSITY OF KYOTO

\section{COMPACT KAEHLER MANIFOLDS WITH POSITIVE RICCI TENSOR}

\author{
BY SHOSHICHI KOBAYASHI
}

Communicated by I. M. Singer, March 6, 1961

The purpose of the present note is to announce the following:

Theorem 1. A compact Kaehler manifold with positive definite Ricci tensor is simply connected.

We say that the first Chern class of a compact Kaehler manifold is positive definite if it can be represented by a real closed $(1,1)$-form which is positive in the sense of Kodaira [2]. The first Chern class of a manifold satisfying the assumption in Theorem 1 is necessarily positive definite. Theorem 1 follows from the following two theorems.

THEOREM 2. If the first Chern class of a compact Kaehler manifold $M$ is positive definite, then the fundamental group of $M$ has no proper subgroup of finite index.

THEOREM OF Myers. The fundamental group of a compact Riemannian manifold with positive definite Ricci tensor is finite [3].

Theorem 2 can be proved by Kodaira's Vanishing Theorem and by the Riemann-Roch Theorem of Hirzebruch. Let $g_{p}$ be the dimension of the space of holomorphic $p$-forms on $M$. Then $\chi(M)=\sum_{p=0}^{n}(-1)^{p} g_{p}$, where $n=\operatorname{dim}_{C} M$, is called the arithmetic genus of $M$. If $M$ is 
algebraic, then $\chi(M)$ is given as the integral over $M$ of a polynomial in Chern classes $c_{i}$ of weight $n$, polynomial depending only on $n$, not on $M$ [1]. From this follows that if $M^{*}$ is a $k$-fold covering space of $M$, then $\chi\left(M^{*}\right)=k \cdot \chi(M)$. On the other hand, if the first Chern class is positive definite, then $g_{p}=0$ for $1 \leqq p \leqq n[2]$ and, hence, the arithmetic genus is 1 . If the first Chern class of $M$ is positive definite, so is the first Chern class of $M^{*}$. Hence, $\chi\left(M^{*}\right)=\chi(M)=1$, proving that $k=1$.

Note that Theorem 2 can be rephrased as follows. If the first Chern class of $M$ is positive definite, then every holomorphic transformation of finite period has fixed points.

In view of the fact that we know no example of a compact Kaehler manifold with positive definite first Chern class whose Ricci tensor is not positive definite, we conjecture that $M$ is simply connected under the assumption of Theorem 2 .

\section{REFERENCES}

1. F. Hirzebruch, Neue topologische Methoden in der algebraischen Geometrie, Ergebnisse der Mathematik und ihrer Grenzgebiete, vol. 9, Berlin, Springer, 1956.

2. K. Kodaira, On a differential-geometric method in the theory of analytic stacks, Proc. Nat. Acad. Sci. U.S.A. vol. 39 (1953) pp. 1268-1273.

3. S. B. Myers, Riemannian manifolds with positive mean curvature, Duke Math. J. vol. 8 (1941) pp. 401-404.

University of British Columbia, Vancouver, B. C., Canada 